and physicians' compliance with the alerts was assessed by measuring the appropriateness of the level 1 interruptive overrides.

Results A total of $42,883 \mathrm{CDA}$ alerts were fired in the adult ICUs: $7.5 \%$ of alerts were severity level 1 (indicates a major severity alert), $20.70 \%$ were level 2 (indicates a moderate severity alert); and $71.8 \%$ were level 3 (indicates a minor severity alert). A total of 3200 overridden major severity alters (level 1) were included for evaluation of physician compliance. An overall appropriateness rate for overridden alerts was $49.9 \%$ and the significance varied by alert category (drug allergy: 66.7\%; drug- drug interactions: 59.7\%; drug disease: 55.4\%; drug dose screening: 29\%).

Conclusion Almost more than $80 \%$ of the CDS alerts were warned of potential significance in patient harm and others had little clinical impact. However, almost 50.1\% were inappropriately overridden and further efforts modification should be focused to improve the CDS alert system, and an uninformative alert must turn off. A future investigation is important to assess why physicians have a low adherence rate for following the recommendations of CDS alerts.

\section{INCIDENCE OF VENTILATOR-ASSOCIATED PNEUMONIA (VAP) IN A TERTIARY- CARE CENTER: COMPARISON BETWEEN THE PRE- AND POST-VAP PREVENTION BUNDLE}

Yousef Al Talhi, Sara Osman, Mona AlDabbagh, Mohamed Baksh, Mohamed Osman, Maha Azzam. College of Medicine, Jeddah, King Saud bin Abdulaziz University for Health Sciences

\subsection{6/bmjoq-2019-PSF.20}

Background Ventilator-associated pneumonia (VAP) is a nosocomial infection that develops 48 hours after the initiation of mechanical ventilatory support. Current evidence-based guidelines demonstrate that VAP prevention is feasible through the simultaneous implementation of certain VAP prevention bundle interventions. In this study we aimed to investigate the effect of VAP prevention before and after implementation.

Methods This was a single-center cohort study that took place at the Pediatric Intensive Care Unit (PICU) of King Abdulaziz Medical City (KAMC), Jeddah, Saudi Arabia, from March 2015 to March 2018, and assessed the rate of VAP before and after implementation of the bundle.

Results The study included 141 children, of whom 95 were included in the pre-bundle group and 36 in the bundle group. VAP developed in $35 \%$ of the pre-bundle group compared with $31 \%$ of the bundle group, with incidence rates of 18 and 12 per 1000 ventilator-days, respectively. Multivariate logistic regression found that high positive end-expiratory pressure (PEEP), high fever (more than $38^{\circ} \mathrm{C}$ ), and high white blood cell count were significant indicators of VAP in our patient population.

Conclusion This study found that the VAP bundle did not significantly reduce VAP rate in the PICU. Further large prospective multicenter studies with longer duration of intervention are needed to investigate the benefits of VAP prevention bundle use.

\section{ANTIMICROBIAL APPROPRIATENESS EVALUATION BASED ON A PROSPECTIVE AUDIT AND FEEDBACK SERVICE LED BY THE ANTIMICROBIAL STEWARDSHIP PROGRAM AT KING ABDULAZIZ MEDICAL CITY - WESTERN REGION}

Nour Shamas, Alaa Al Juaid, Maher Al Harbi, Mohammad Aseeri, Asim Al Saedi. Infection Prevention and Control King Abdulaziz Medical City - WR

\subsection{6/bmjoq-2019-PSF.21}

Background The Antimicrobial Stewardship Program (ASP) at King Abdulaziz Medical City - Western Region (KAMC-WR), initiated in November 2016, aims to improve the use of antimicrobials at our facility. It is internationally recognized that one of the cornerstones of ASP is prospective audit and feedback (PAF). In an effort to improve antimicrobial use in areas at high risk for antimicrobial resistance, the ASP initiated a PAF service in February 2018 at the pediatric intensive care unit (ICU), pediatric cardiac ICU, adult ICU, and adult hematology-oncology ward. This retrospective study describes PAF interventions and antimicrobial appropriateness based on PAF.

Methods An automatically generated daily antimicrobial report built by the ASP and ISD team is used by the PAF to assess patients not under infectious disease (ID) consultation on prespecified antimicrobials for appropriateness of indication, dose, route, and frequency. The PAF service is run by the PICU or ASP/ID clinical pharmacist, ID fellow, and ID consultant. The PAF teams meet with the respective departments for discussion and communication of recommendations twice weekly for pediatric patients and daily for adults. A customized PAF electronic note is filled for each order. The audited antimicrobials are imipenem, meropenem, colistin, tigecycline, linezolid, vancomycin IV, anidulafungin, caspofungin, and voriconazole. An electronic report of the PAF note is generated regularly by the ISD team to assess PAF. Simple descriptive statistics were used for the analysis.

Results A total of 747 PAF consultations were documented from February 2018 to February 2019, 92\% of which were in adults (686 of 747). 35\% of all consultations were inappropriate. Of the 259 inappropriate medication orders, most were in adults (97\%) with pneumonia (40\%) and sepsis (27\%). $68 \%$ of inappropriate orders were empiric, and the rest had no clear indication or were therapeutic. The most common inappropriate element of adult orders was choice of antimicrobial, followed by dose, frequency, and then route $(83 \%, 6 \%, 4 \%$, and $1 \%$, respectively). All inappropriate pediatric orders were inappropriate choices. For adults, meropenem and vancomycin consisted of $67 \%$ of inappropriate choices of antimicrobial (140 of 208). Of all antimicrobials, tigecycline had the highest rate of inappropriate choice (10 of 21 ).

Conclusion This study shows that antimicrobial use guidelines for meropenem, vancomycin, and tigecycline need to be reevaluated and reinforced through continuous PAF and the creation of clinical practice guidelines with electronic order sets for pneumonia and sepsis. The results of this workflow embedded electronic assessment will help the ASP at KAMCWR tailor future interventions that promote safe and effective antimicrobial use. 